\title{
Changes in cosmic ray cut-off rigidities due to secular variations of the geomagnetic field
}

\author{
A. Bhattacharyya ${ }^{1}$, B. Mitra $^{2}$ \\ ${ }^{1}$ Indian Institute of Geomagnetism, Colaba, Bombay 400005, India \\ ${ }^{2}$ Department of Physics, Jadavpur University, Calcutta, India
}

Received: 13 January 1996 / Revised: 14 January 1997 / Accepted: 17 February 1997

\begin{abstract}
An analytical expression is derived for the cutoff rigidity of cosmic rays arriving at a point in an arbitrary direction, when the main geomagnetic field is approximated by that of an eccentric dipole. This expression is used to determine changes in geomagnetic cutoffs due to secular variation of the geomagnetic field since 1835. Effects of westward drift of the quadrupole field and decrease in the effective dipole moment are seen in the isorigidity contours. On account of the immense computer time required to determine the cutoff rigidities more accurately using the particle trajectory tracing technique, the present formulation may be useful in estimating the transmission factor of the geomagnetic field in cosmic ray studies, modulation of cosmogenic isotope production by geomagnetic secular variation, and the contribution of geomagnetic field variation to long term changes in climate through cosmic ray related modulation of the current flow in the global electric circuit.
\end{abstract}

\section{Introduction}

The Störmer formula (1955) for cosmic ray cutoff rigidity, which assumed the geomagnetic field to arise due to a dipole located at the centre of the Earth, has been widely used in many studies. A more accurate description of the main geomagnetic field is the one derived from a scalar potential, which arises due to sources internal to the Earth and is represented in terms of a spherical harmonic expansion involving multipoles (Langel, 1987 and references therein):

$\vec{B}=-\vec{\nabla} V$

Correspondence to: A. Bhattacharyya

$$
V=a \sum_{n=1}^{N} \sum_{m=0}^{n}\left[g_{n}^{m} \cos m \phi+h_{n}^{m} \sin m \phi\right]\left(\frac{a}{r}\right)^{n+1} P_{n}^{m}(\cos \theta)
$$

Here, $a$ is the mean radius of the Earth's surface, $r, \theta$, and $\phi$ are spherical coordinates with origin at the centre of the Earth, such that $\theta$ is the co-latitude and $\phi$ is the longitude, and $g_{n}^{m}, h_{n}^{m}$ are coefficients which are determined from observations. There does not exist an exact closed form expression for cosmic ray cutoff rigidity for an arbitrary angle of arrival at an arbitrary location when higher order terms beyond the dipole $(n=1)$ are retained in the spherical harmonic expansion, (2). In this situation, the most direct method for computing cutoff rigidities in the geomagnetic field is to trace the trajectories of individual particles as they move through the geomagnetic field (Kellogg, 1960; McCracken, 1962). The drawback of this method is the enormous amount of computer time required to obtain the vertical cutoff rigidities at enough locations to arrive at a picture of the isorigidity contours, or to compute cutoff rigidities for various directions of incidence. The problem has also been approached in a different way in which corrections to the Störmer integral were determined, to take into account effects of higher order $(n>1)$ terms in the spherical harmonic models of the geomagnetic field (Quenby and Webber, 1959). These authors derived corrections for the cutoff rigidity in the equatorial region, which resulted in considerable improvement over the Störmer cutoff in this region. Starting with the centred dipole model, Quenby and Wenk (1962) determined corrections to the cutoff rigidities in three latitude regions by three methods. For geomagnetic latitudes below $55^{\circ}$ corrections were applied for penumbral effects, arising due to the finite dimensions of the Earth, which led to better agreement with observations. In another approach, first put forth by Ray (1963), the Euler potential representation of the geomagnetic field was utilized to obtain improved cutoff rigidities in high-latitude regions where the cutoff rigidities are low. 
For some applications, it is necessary to derive the dependence of the cutoff rigidity on the direction of incidence of the particle at any location (Masarik and Reedy, 1995). Bland and Cioni (1968) have computed geomagnetic cutoff rigidities in non-vertical directions using an expresssion for the cutoff rigidity derived from a first integral analogous to the Störmer integral, obtained for an Euler potential representation of the geomagnetic field (Ray, 1963). A special case of this yields the vertical cutoffs derived from McIlwain's $L$ parameter (1961) discussed later in the work. However the basic theory used in the derivation is not applicable at high rigidity values. Thus this method is useful for obtaining improved cutoffs at high latitudes. In the search for satisfactory results for cutoff rigidities obtained without recourse to large scale computations, Smart and Shea (1995) introduced the offset dipole coordinates and expanded the Störmer expresssion for vertical cutoff around the location of the eccentric dipole representing the geomagnetic field, to obtain approximate cutoff values at the top of the Earth's atmosphere. This treatment did not take into account properly the direction of incidence of the cosmic rays at a certain location with reference to the eccentric dipole but it did introduce longitudinal asymmetry in the cutoff rigidities due to the displacement of the effective dipole from the centre of the Earth. However, there was a large longitudinal discrepancy in the position of the maximum cutoff contour when compared to cutoff rigidity contours derived from cosmic ray trajectory tracing. Although this shortcoming of the eccentric dipole approximation for the geomagnetic field, as far as rigidity calculations are concerned, has been known for a long time, the only justification for employing this approximation in the present study is that in this case an exact analytical expression could be derived for the cutoff rigidity for arbitrary direction of incidence of the cosmic ray particles. Thus, the process of obtaining global averages, when different directions of incidence are involved, could be considerably simplified. This formulation can be easily used to study the changes in isorigidity contours of cosmic ray cutoff rigidities due to variation in the location and strength of the eccentric dipole in the recent past. In comparison, the computer codes used to calculate effective cutoff rigidities by trajectory tracing techniques, which yield a more accurate picture of the isorigidity contours by taking into consideration spatial variations of the geomagnetic field on much shorter scale length $(\sim 1000 \mathrm{~km}$ at the surface of the Earth) as also the finite size of the Earth, require enormous computer time. Hence, it may not be feasible, in practice, to use such techniques to study the changes in geomagnetic cutoffs due to secular variation of the geomagnetic field (Shea and Smart, 1970).

\section{Cutoff rigidity in the field of an eccentric dipole}

In this section an expression for the cutoff rigidity is derived for particles arriving at a particular location from an arbitrary direction. The starting point of the derivation is the Störmer equation (1955) for the cutoff rigidity $P_{c}$, for arbitrary angle of incidence of the particle at a distance $R$ from the centre of the dipole:

$P_{c}=\frac{M}{R^{2}} \frac{\cos ^{4} \Psi}{\left[1+\left(1-\sin \alpha \cos ^{3} \Psi\right)^{1 / 2}\right]^{2}}$

where $M$ is the magnetic moment of the dipole, the angle $\Psi$ equals $\left(90^{\circ}-\Phi\right), \Phi$ being the angle between the dipole axis and the vector $\vec{R}$ joining the centre of the dipole and the point of incidence, and $\alpha$ is the angle that the particle trajectory makes with the plane containing the dipole and the point of incidence $P$ (Fig. 1), considered positive if a positively charged particle crosses this plane from east to west. If the geomagnetic field is considered to be that due to an eccentric dipole, and one wants to calculate the cutoff rigidity at a fixed altitude above the surface of the Earth, the distance $R$ of the point of incidence from the centre of this dipole varies with latitude and longitude of the point $P$.

Location of the 'best-fit' eccentric dipole is obtained from the terms upto $n=2$ in the spherical harmonic representation of the geomagnetic field (James and Winch, 1967; Langel, 1987). In a cartesian coordinate system with origin at Earth's centre $O, z$-axis along the Earth's rotation axis, and $x$-axis in the equatorial plane and Greenwich meridian, the $(x, y, z)$ coordinates of $C$, the position of the eccentric dipole, are given by:

$$
\begin{aligned}
X_{0} & =a\left(L_{1}-g_{1}^{1} T\right) / 3 m^{2} \\
Y_{0} & =a\left(L_{2}-h_{1}^{1} T\right) / 3 m^{2} \\
Z_{0} & =a\left(L_{0}-g_{1}^{0} T\right) / 3 m^{2}
\end{aligned}
$$

where $L_{0}, L_{1}, L_{2}, T$, and $m$ are derived from the coefficients $g_{n}^{m}$ and $h_{n}^{m}$ as follows:

$$
\begin{aligned}
& m^{2}=\left(g_{1}^{0}\right)^{2}+\left(g_{1}^{1}\right)^{2}+\left(h_{1}^{1}\right)^{2} \\
& L_{0}=2 g_{2}^{0} g_{1}^{0}+\sqrt{3}\left(g_{2}^{1} g_{1}^{1}+h_{2}^{1} h_{1}^{1}\right) \\
& L_{1}=-g_{2}^{0} g_{1}^{1}+\sqrt{3}\left(g_{2}^{1} g_{1}^{0}+g_{2}^{2} g_{1}^{1}+h_{2}^{2} h_{1}^{1}\right)
\end{aligned}
$$

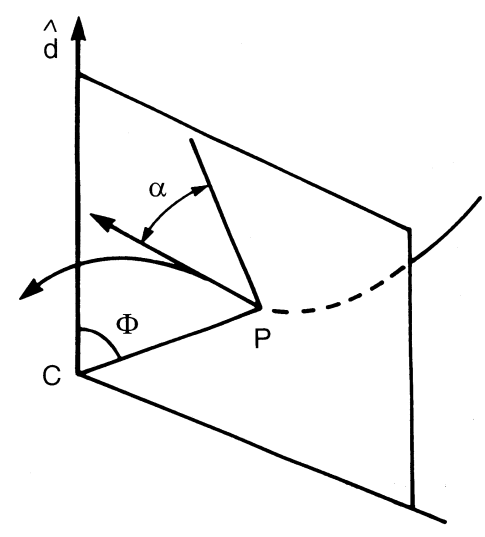

Fig. 1. Definition of $\alpha$ as the angle between the plane containing the dipole and the point of incidence $P$, and the particle trajectory at $P$ 


$$
\begin{aligned}
& L_{2}=-g_{2}^{0} h_{1}^{1}+\sqrt{3}\left(h_{2}^{1} g_{1}^{0}+h_{2}^{2} g_{1}^{1}-g_{2}^{2} h_{1}^{1}\right) \\
& T=\frac{\left(L_{0} g_{1}^{0}+L_{1} g_{1}^{1}+L_{2} h_{1}^{1}\right)}{4 m^{2}}
\end{aligned}
$$

It is to be noted that if $g_{2}^{0}, g_{2}^{1}, h_{2}^{1}, g_{2}^{2}$ and $h_{2}^{2}$ are all zero, then $L_{0}=0, L_{1}=0, L_{2}=0$, and $T=0$. Hence in this case, $X_{0}=0, Y_{0}=0, Z_{0}=0$; i.e. $C$ coincides with $O$, the centre of the Earth, yielding a centred dipole.

Suppose the geocentric co-latitude and longitude of the point of incidence $P$ at a distance $r$ from the centre of the Earth are $\delta$ and $\lambda$ such that its $(x, y, z)$ coordinates are:

$$
\begin{aligned}
X & =r \sin \delta \cos \lambda \\
Y & =r \sin \delta \sin \lambda \\
Z & =r \cos \delta
\end{aligned}
$$

Then $\vec{R}$ is given by:

$\vec{R}=\left(X-X_{0}\right) \hat{x}+\left(Y-Y_{0}\right) \hat{y}+\left(Z-Z_{0}\right) \hat{z}$

$\hat{x}, \hat{y}$, and $\hat{z}$ being unit vectors along the $x$-, $y$ - and $z$-axes. A unit vector, $\hat{d}$, along the direction of the dipole axis is parallel to the tilted, centred dipole axis; which is in a direction specified by colatitude $\theta_{0}$ and longitude $\phi_{0}$ determined by (James and Winch, 1967):

$\sin \theta_{0}=\frac{\left[\left(g_{1}^{1}\right)^{2}+\left(h_{1}^{1}\right)^{2}\right]^{1 / 2}}{m}, \quad \tan \phi_{0}=\frac{h_{1}^{1}}{g_{1}^{1}}$

Thus

$\hat{d}=\sin \theta_{0} \cos \phi_{0} \hat{x}+\sin \theta_{0} \sin \phi_{0} \hat{y}+\cos \theta_{0} \hat{z}$

Since $\Phi$ is the angle between $\vec{R}$ and $\hat{d}, \cos \Phi$ can be obtained from:

$$
\begin{aligned}
\cos \Phi= & \frac{\vec{R} \cdot \hat{d}}{R} \\
= & {\left[\left(X-X_{0}\right) \sin \theta_{0} \cos \phi_{0}+\left(Y-Y_{0}\right) \sin \theta_{0} \sin \phi_{0}\right.} \\
& \left.\quad+\left(Z-Z_{0}\right) \cos \theta_{0}\right] / R
\end{aligned}
$$

Hence $\cos \boldsymbol{\Psi}$, which is required in Eq. (3) can be calculated from

$$
\cos \boldsymbol{\Psi}=\left(1-\cos ^{2} \Phi\right)^{1 / 2}
$$

In order to determine $\sin \alpha$ which is also required in Eq. (3), use is made of the relation

$\sin \alpha=\cos \omega$

where $\omega$ is the angle between the particle trajectory at the point of incidence $P$, and the normal to the meridional plane containing the dipole and the point $P$. Since the vectors $\hat{d}$ and $\vec{R}$ define this meridional plane, $\vec{R} \times \hat{d}$ is directed along the normal to this plane. If the particle arrives at $P$ at an angle $\xi$ from the zenith and azimuthal angle $\eta$, measured counter-clockwise from the local east, its trajectory is along the unit vector $\hat{t}$ :

$\hat{t}=t_{x} \hat{x}+t_{y} \hat{y}+t_{z} \hat{z}$

where

$$
\begin{aligned}
t_{z}= & -\cos \xi \sin \delta \cos \lambda+\sin \xi \sin \eta \cos \delta \cos \lambda \\
& +\sin \xi \cos \eta \sin \lambda \\
t_{y}= & -\cos \xi \sin \delta \sin \lambda+\sin \xi \sin \eta \cos \delta \sin \lambda \\
& -\sin \xi \cos \eta \cos \lambda \\
t_{z}= & -\cos \xi \cos \delta-\sin \xi \sin \eta \sin \delta
\end{aligned}
$$

As $\omega$ is the angle between the vectors $\hat{t}$ and $\vec{R} \times \hat{d}, \cos \omega$ can be obtained from:

$\cos \omega=\frac{\hat{t} \cdot(\vec{R} \times \hat{d})}{|\vec{R} \times \hat{d}|}$

According to Eqs. (11) and (13), $\vec{R} \times \hat{d}$ has the form:

$$
\begin{aligned}
\vec{R} \times \hat{d}= & {\left[\left(Y-Y_{0}\right) \cos \theta_{0}-\left(Z-Z_{0}\right) \sin \theta_{0} \sin \phi_{0}\right] \hat{x} } \\
& +\left[\left(Z-Z_{0}\right) \sin \theta_{0} \cos \phi_{0}-\left(X-X_{0}\right) \cos \theta_{0}\right] \hat{y} \\
& +\left[\left(X-X_{0}\right) \sin \theta_{0} \sin \phi_{0}\right. \\
& \left.-\left(Y-Y_{0}\right) \sin \theta_{0} \cos \phi_{0}\right] \hat{z} .
\end{aligned}
$$

using which $\sin \alpha$ can be computed on the basis of Eqs. (16) and (21). Since the magnetic moment $M$ in Störmer's formula (3) is given in terms of $m$ by

$M=m a^{3}$

where $m$ itself is obtained in terms of $g_{1}^{0}, g_{1}^{1}$ and $h_{1}^{1}$ from Eq. (5), the cutoff rigidity at the point $P$ can be calculated for the eccentric dipole using Eq. (3) itself, with $\sin \alpha$ now given by Eq. (21) and $\cos \Psi$ given by Eqs. (14) and (15).

As mentioned already, the result of retaining only the $n=1$ term in the spherical harmonic expansion for the geomagnetic field, is a centered, tilted dipole. If the $z$ axis is rotated to coincide with the dipole axis of this centred, tilted dipole, then $\sin \alpha$ reduces to

$$
\sin \alpha=\sin \xi \cos \eta
$$

as it should in this case, and $\Psi$ is simply the dipole latitude of the point of incidence $P$, so that the Störmer formula is recovered.

\section{Vertical cosmic ray cutoff rigidity}

In the particular situation where the particle is vertically incident at $P$, the trajectory is directed along $-\vec{r}$ and $\hat{t}=-\vec{r} / r$. In this case, $\sin \alpha$ is given by:

$$
\begin{aligned}
\sin \alpha=[ & \left(Z X_{0}-X Z_{0}\right) \sin \theta_{0} \sin \phi_{0}+\left(X Y_{0}-Y X_{0}\right) \cos \theta_{0} \\
& \left.+\left(Y Z_{0}-Z Y_{0}\right) \sin \theta_{0} \cos \phi_{0}\right] / r|\vec{R} \times \hat{d}|
\end{aligned}
$$

Vertical cosmic ray cutoff rigidities, at an altitude of $100 \mathrm{~km}$ above a spherical Earth, were computed on the basis of these equations using the geomagnetic field coefficients $g_{n}^{m}, h_{n}^{m}$ upto $n=2$. Results obtained for the vertical cosmic ray effective cutoff rigidities by using a computer code for tracing the trajectories of individual particles (Shea et al., 1965) in the geomagnetic field described by the IGRF (International Geomagnetic Reference Field) coefficients upto degree $n=10$, for epoch 1985 (Langel, 1987), are shown in Fig. 2. This 


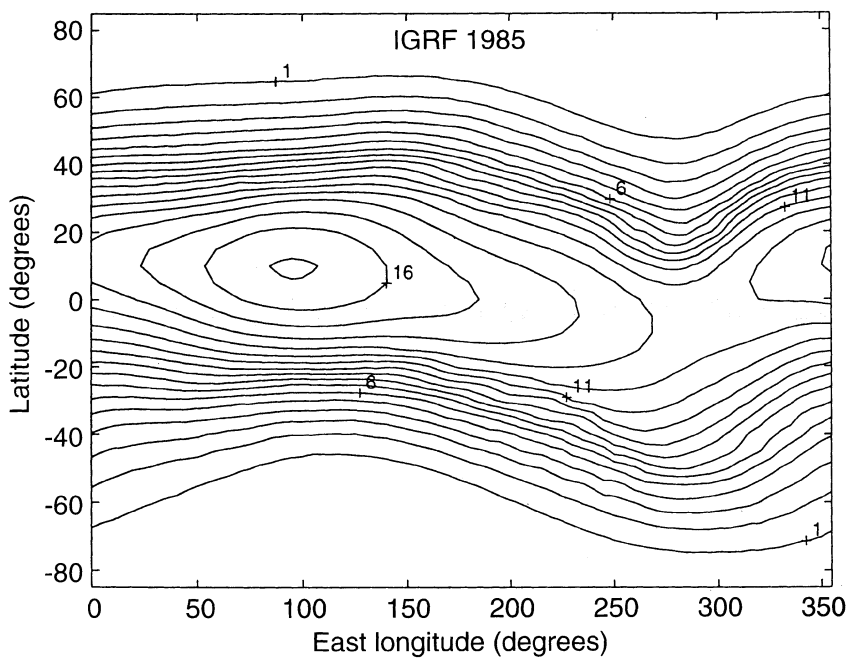

Fig. 2. Isorigidity contours of vertical cosmic ray effective cutoff rigidities (in GV), at an altitude of $100 \mathrm{~km}$, computed by tracing trajectories in the geomagnetic field represented by the IGRF model for epoch 1985 with maximum $n=10$ figure is to be compared with Fig. 3d, which shows contours of vertical cosmic ray cutoff rigidity (in GV), calculated as described in the field of the best-fit eccentric dipole obtained from IGRF1985. In this calculation, unlike the more accurate trajectory tracing technique, no account has been taken of the penumbra in rigidity due to the finite dimensions of the Earth. However, for proper comparison with results obtained by tracing individual trajectories, the actual shape of the Earth has been considered in converting geodetic coordinates to geocentric ones. Thus the latitudes in Fig. 3, as in Fig. 2, are geographic latitudes. The major difference between the two figures is the shift in the location of the maximum in vertical cutoff rigidity, by about $30^{\circ}$ in longitude, and around $6 \%$ decrease in the maximum value of the cutoff rigidity for the eccentric dipole, which is found to be nearly $16.6 \mathrm{GV}$. In some regions, the eccentric dipole approximation gives rise to errors of the order of $20 \%$. Nevertheless, a major advantage of the present generalisation of the Störmer equation is that it allows a quick calculation of cutoff
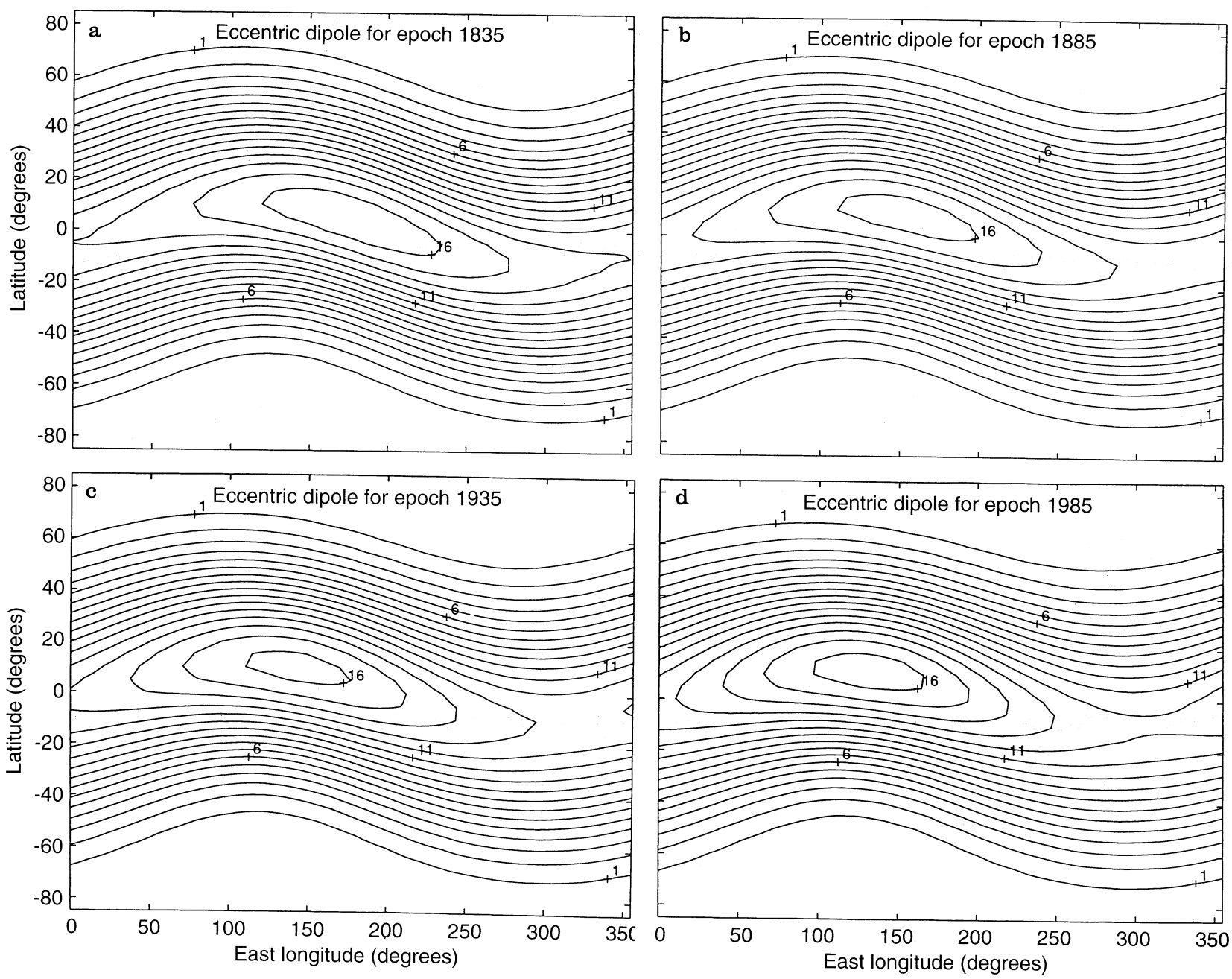

Fig. 3a-d. Isorigidity contours of vertical cosmic ray cutoff rigidities (in GV), at an altitude of $100 \mathrm{~km}$, computed in the geomagnetic field approximated by an eccentric dipole, for epochs 1835, 1885, 1935, and 1985 
rigidities for all directions of incidence of cosmic rays at all points, and also yields results which are a considerable improvement over the results derived from the Störmer formula. Apart from the exclusion of penumbral effects, the present formulation for cutoff rigidities in the field of an eccentric dipole does not involve any other approximation, even for non-vertical directions, unlike the results obtained by Smart and Shea (1995) or those based on McIlwain's $L$ parameter.

As an example of the application of the present formulation, changes in vertical cosmic ray rigidity cutoffs due to variation in both the location of the eccentric dipole, as well as the strength of its dipole moment, since 1835 , are computed. Contours of vertical cutoff rigidities calculated using geomagnetic field models approxmiated by an eccentric dipole for epochs 1835, 1885, 1935 and 1985 are shown in Fig. 3. Barraclough (1978) has described the first three of these models. For 1985, the IGRF model (Langle, 1987) has been used, as mentioned earlier. Prior to 1800 , only declination data were available from about 1550 , with inclination data being added around 1700. Consequently, only the ratios $g_{n}^{m} / g_{1}^{0}$ and $h_{n}^{m} / g_{1}^{0}$ could be determined upto 1800 . The coefficient $g_{1}^{0}$, and hence the strength of the effective dipole moment of the Earth, could be accurately determined only after measurements of local geomagnetic field intensity were made during the nineteenth century. These estimates indicate an almost steady decrease in the dipole moment since 1800 (Langel, 1987). The effect of this decrease in the dipole moment on vertical cutoff rigidities is most pronounced in the equatorial region where the geomagnetic field lines are nearly horizontal. In this region, the vertical cutoff rigidities decrease with time, which results in a progressive shrinking of areas with high cutoff rigidity from 1835 to 1985 as seen in Fig. 3. The other notable feature is the westward drift of the location of maximum cutoff at an average rate of approximately $0.3^{\circ}$ per year over the past 150 years. This is expected as the main geomagnetic field shows a general westward drift of this order during the past couple of centuries (Langel, 1987), and the contribution of a westward drifting quadrupole field towards this general westward drift is significant.

\section{Conclusion}

It has long been recognised that secular variation of the geomagnetic field contributes to significant changes in the production rate of radiocarbon and other cosmogenic isotopes (Damon et al., 1978). Assuming the geomagnetic field to be that due to a centred dipole, Elsasser et al. (1956) and later Castagnoli and Lal (1980), have used the Störmer cutoff rigidity to estimate the changes in the production rate of radiocarbon due to variation of the dipole moment. However, the effect of variations in the non-dipole part of the geomagnetic field on the incoming cosmic ray flux also needs to be studied in order to examine the possibility, raised by Creer (1988), of the persistent presence of a strong drifting quadrupole field maintaining a small axially asymmetric input of ${ }^{14} C$ against atmospheric mixing. After ${ }^{14} C$ is produced by cosmic ray particles impinging on the atmosphere, it is oxidised and exchanged between atmosphere, ocean and biosphere. To test Creer's (1988) idea, it would be necessary to introduce an appropriate carbon cycle model inorder to obtain a quantitative estimate of the effect of secular variation in the eccentric dipole on atmospheric ${ }^{14} C$. Hence it is beyond the scope of the present work to demonstrate the extent to which non-inclusion of terms beyond the quadrupole in the description of the geomagnetic field, and penumbral effects, will change the long term $\left(>10^{2} \mathrm{y}\right)$ variations in atmospheric ${ }^{14} C$.

In a recent paper, Smart and Shea (1993) discussed the possible use of the McIlwain ' $L$ ' parameter (McIlwain, 1961) to estimate the geomagnetic cutoffs inorder to study the changes in these cutoffs due to geomagnetic field variations. However, as these authors have themselves pointed out, the ' $L$ ' parameter is inappropriate at equatorial latitudes where the value of the ' $L$ ' parameter for a low altitude point may be less than 1 . In fact, it is seen that the vertical cutoff rigidities derived using $L$-values less than 1.5 are less accurate than the cutoff rigidities obtained by using the present formulation for the eccentric dipole. Thus, the ' $L$ ' parameter may be more useful for geographical latitudes above $30^{\circ}$ (Shea et al., 1987). At lower latitudes the present generalisation of the Störmer formula, to include quadrupole terms in the spherical harmonic expansion for the geomagnetic field, may be used to estimate changes in the geomagnetic cutoffs. This method goes a step beyond the centred dipole approximation, which has so far been used to study the changes in the production rate of ${ }^{14} C$ due to changes in the geomagnetic field (Castagnoli and Lal, 1980). For a more accurate global picture, it is necessary to resort to tracing of individual particle trajectories. In recent years, time-dependent models of the main geomagnetic field going back to 1690 have been produced (Bloxham and Jackson, 1992), using which, long term $\left(>10^{2} \mathrm{y}\right)$ variations in cutoff rigidities of cosmic rays can be computed. Enhanced cosmic ray production of ${ }^{10} \mathrm{Be}$ coincident with the Mono Lake and Laschamp geomagnetic excursions have been reported in recent literature (McHargue et al., 1995). A mechanism which has been proposed for this enhancement, by these authors, is the change in galactic cosmic ray flux coincident with a supernova event. In order to test such a hypothesis it is necessary to quantify the increase in radioisotope production due to variations in the geomagnetic field as well as in the solar activity modulating the cosmic ray flux. The present formulation can be easily used in a series of model calculations.

Another potential area of application is estimation of the terrestrial modulation of current flow in the global electric circuit due to long term changes in the geomagnetic field. Such a modulation could occur as a result of changes in atmospheric conductivity due to variations in the incoming cosmic ray flux produced by secular variation in the geomagnetic field. The possibi- 
lity of this mechanism being a contributary factor towards the natural variability of climate has been suggested in recent times (Tinsley and Deen, 1991; Tinsley, 1994). In conclusion, those problems which require a less detailed calculation of the changes in cosmic ray flux due to secular variation of the geomagnetic field, than offered by the trajectory tracing method, may benefit from the results presented here.

Acknowledgements. The authors would like to thank M. A. Shea and D. F. Smart for use of their computer code for trajectory tracing in the geomagnetic field.

Topical Editor thanks M. A. Shea, J. J. Quenby and another referee for their help in evaluating this paper.

\section{References}

Barraclough, D. R., Spherical harmonic models of the geomagnetic field, Geomagn. Bull. Inst. Geol. Sci., 8, 1978.

Bland, C. J., and G. Cioni, Geomagnetic cutoff rigidities in nonvertical directions, Earth Planet. Sci. Lett., 4, 399, 1968.

Bloxham, J., and A. Jackson, Time-dependent mapping of the magnetic field at the core-mantle boundary, J. Geophys. Res., 97, 19537, 1992.

Castagnoli, G., and D. Lal, Solar modulation effects in terrestrial production of carbon-14, Radiocarbon, 22, 133, 1980.

Cooke, D. J., J. E. Humble, M. A. Shea, D. F. Smart, N. Lund, I. L. Rasmussen, B. Byrnak, P. Goret and N. Petrou, On cosmic-ray cutoff terminology, Nuovo Cimento, 14, 213, 1991.

Creer, K. M., Geomagnetic field and radiocarbon activity through Holocene time, Secular Solar and Geomagnetic Variations in the Last 10000 Years, Ed. F. R. Stephenson and A. W. Wolfendale, Kluwer Academic Publishers, Dordrecht, The Netherlands, 1988.

Damon, P. E., J. C. Lerman, and A. Long, Temporal fluctuations of atmospheric ${ }^{14} \mathrm{C}$ : causal factors and implications, Ann. Rev. Earth Planet. Sci., 6, 457, 1978.

Elsasser, W., E. P. Ney and J. R. Winckler, Cosmic ray intensity and geomagnetism, Nature, 178, 1226, 1956.

James, R. W. and D. E. Winch, The eccentric dipole, Pure Appl. Geophys., 66, 77, 1967.
Kellogg, P. J., Calculations of cosmic-ray trajectories near the equator, J. Geophys. Res., 65, 2701, 1960.

Langel, R. A., The main field, in Geomagnetism, 1, Ed. J. A. Jacobs, Academic Press, London, 1987.

Masarik, J., and R. C. Reedy, Terrestrial cosmogenic-nuclide production systematics calculated from numerical simulations, Earth Planet. Sci. Lett., 136, 381, 1995.

McCracken, K. G., The cosmic-ray flare effect, I. Some new methods of analysis, J. Geophys. Res., 67, 423, 1962.

McHargue, L. R., P. E. Damon and D. J. Donahue, Enhanced cosmic-ray production of ${ }^{10} \mathrm{Be}$ coincident with the Mono Lake and Laschamp geomagnetic excursions, Geophys. Res. Lett., 22, 659, 1995.

Mcllwain, C. E., Coordinates for mapping the distribution of magnetically trapped particles, J. Geophys. Res., 66, 3681, 1961.

Quenby J. J. and W. R. Webber, Cosmic ray cutoff rigidities and the earth magnetic field, Philos. Mag., 4, 90, 1959.

Quenby, J. J. and G. J. Wenk, Cosmic ray threshold rigidities and the earth's magnetic field, Philos. Mag., 7, 1457, 1962.

Ray, E. C., On the motion of charged particles in the geomagnetic field, Ann. Phys., 24, 1, 1963.

Shea, M. A., D. F. Smart, Secular variations in cosmic ray cutoff rigidities, J. Geophys. Res., 75, 3921, 1970.

Shea, M. A., D. F. Smart, and K. G. McCracken, A study of vertical cutoff rigidities using sixth degree simulations of the geomagnetic field, J. Geophys. Res., 70, 4117, 1965.

Shea, M. A., D. F. Smart, and L. C. Gentile, Estimating cosmic-ray vertical cutoff rigidities as a function of the Mcllwain LParameter for different epochs of the geomagnetic field, Phys. Earth Planet. Inter., 48, 200, 1987.

Smart, D. F. and M. A. Shea, The change in geomagnetic cutoffs due to changes in the dipole equivalent of the Earth's magnetic field, $23^{\text {rd }}$ International Cosmic Ray Conference Papers, 3, 781, 1993.

Smart, D. F. and M. A. Shea, The difference between offset dipole coordinates and coordinates based on cutoff rigidity contours, $24^{\text {th }}$ International Cosmic Ray Conference Papers, 4, 1062, 1995.

Störmer, C., The Polar Aurora, Clarendon Press, Oxford, 1955.

Tinsley, B. A., Solar wind mechanism suggested for weather and climate change, EOS Trans. AGU, 75, 360, 1994.

Tinsley, B. A. and G. W. Deen, Apparent tropospheric response to $\mathrm{MeV}-\mathrm{GeV}$ particle flux variations: a connection via electrofreezing of supercooled water in high-level clouds?, J. Geophys. Res., 96, 22283, 1991. 